ORIGINAL ARTICLE

\title{
Attention and working memory in resident anaesthetists after night duty: group and individual effects
}

\author{
P Bartel, W Offermeier, F Smith, P Becker
}

Occup Environ Med 2004;61:167-170. doi: 10.1136/oem.2002.006197

See end of article for authors' affiliations

.....................

Correspondence to:

Dr P Bartel, Department of Neurology, Pretoria Academic Hospital, Private bag X169, 0001 Pretoria, South Africa; pbartel@ medic.up.ac.za

Accepted 26 March 2003

\begin{abstract}
Aims: To investigate the effects of a single period of night duty on measures of attention and working memory in a group of residents (registrars) in anaesthesiology. Emphasis was placed on individual deficits using a reference point of the equivalent effect of a blood alcohol concentration (BAC) $>0.05 \%$ determined by other researchers.

Methods: There were 33 subjects aged 26-42 years. Night duty was performed on a weekly basis. Baseline assessments were conducted at either 0815 or 0855 preceding night duty and repeated 2425 hours later, just after the completion of duty. Questionnaires included items regarding duration of sleep and the Stanford Sleepiness Scale. A battery of four reaction time (RT) tasks of increasing difficulty, lasting approximately 35 minutes, was administered on a personal computer. These ranged from simple RT to progressively more complex RT tasks incorporating working memory. A significant change was regarded as $>15 \%$ deterioration in respect of speed or accuracy.

Results: The mean duration of sleep preceding night duty was 7.04 hours and 1.66 hours during the period of night duty. Intergroup comparisons revealed significant prolongation in mean response speed in the first three tests. Mean accuracy was significantly reduced only in respect of the two more complex tests. A $>15 \%$ deterioration in response speed occurred in up to $30 \%$ of subjects on a single task, rising to $52 \%$ $(17 / 33)$ overall. Deterioration occurred in a patchy distribution in most subjects, involving no more than one or two of the four tasks. As regards accuracy, the prevalence of deterioration increased with task complexity.

Conclusions: Results are in general agreement with previous group analyses. A new dimension was added by the analysis of a broad spectrum of individual response to sleep deprivation. The effects of sleep loss in residents cannot be overlooked, even in a relatively benign work schedule.
\end{abstract}

artial sleep deprivation, of the degree commonly experienced by on-call residents, impairs cognitive functioning. ${ }^{1}$ The practical significance of these changes continues to be debated. ${ }^{2}{ }^{3}$ Ideally, objective changes in the clinical capabilities of sleep deprived doctors would inform the debate. These assessments are difficult, and therefore neuropsychological tests have served as proxy measures in many studies. ${ }^{4}$

The relevance of laboratory based tests to real world tasks has been questioned. ${ }^{5}$ Nonetheless reaction time (RT) tasks would seem to have some face validity for medical staff where monitoring of electronic equipment is important. However, studies in the period 1970-90 revealed inconsistent results. There was a general trend for short duration tests of reaction time (RT) to fail to detect significant decline in performance after sleep loss of one night. ${ }^{4} \mathrm{~A}$ recent review contributed few additional RT studies. ${ }^{3}$

An assessment of choice RT in a group of junior anaesthetists at three sequential times during a period of on-call duty failed to detect significant deterioration between the afternoon and 0200 the following morning. ${ }^{6}$ Using a longer test battery, Orton and Gruzelier ${ }^{7}$ found significant deterioration in RT in a vigilance task but not in a simpler choice RT task. Using a similar range of tests, subjects were assessed after a weekend off duty and after a weekend that included duty periods of approximately 35 hours with minimal sleep. An overall score of attention speed showed a significant deterioration following a weekend on-call. ${ }^{8}$

Recently, a new yardstick has emerged to establish the importance of changes in performance, particularly those related to sleep deprivation. After 17 hours of sustained wakefulness, hand-eye coordination was reported to deteriorate to a level achieved by a blood alcohol concentration (BAC) of $0.05 \%{ }^{9}$ This equates to an $8-15 \%$ decline in a range of various performance measures, including simple RT. ${ }^{10}$

We used this criterion to evaluate individual resident anaesthesiologists after a single session of night duty, an approach not previously reported. The pitfall of short duration tests was avoided by using a battery of four RT tasks of increasing complexity to assess attention and working memory. Each test exceeded the five minute level regarded by Richardson and colleagues ${ }^{11}$ as necessary to detect sleep deprivation effects.

\section{SUBJECTS AND METHODS}

Approval was obtained from the relevant institutional ethics committee. The subjects were 33 residents in anaesthesiology who gave written informed consent to participate. There were 17 males and 16 females (age range 26-42 years, mean age 32 years). Night duty commenced at 1600 on an otherwise offduty day allocated to study. The duty period ended at 0800 the following morning. This type of duty was performed once a week. The mean number of hours worked per week was 60.5 (range 55-67 hours).

The baseline assessment was conducted at either 0815 or 0855 preceding night duty and then repeated 24 hours later after the completion of duty. A questionnaire included items

Abbreviations: $\mathrm{BAC}$, blood alcohol concentration; RT, reaction time; SSS, Stanford Sleepiness Scale 
Main messages

- Important psychomotor performance deficits occurred in $52 \%$ of a group of resident anaesthetists after night duty.

- These deficits occurred intermittently during a test session of approximately 35 minutes compatible with lapses in vigilance.

- While response speed was more affected than response accuracy, as task complexity increased there was an increased occurrence of missed targets or invalid responses to targets.

regarding the usual length of nocturnal sleep and that obtained during the previous night. The Stanford Sleepiness Scale $^{12}$ was then completed. This is a seven point self rating scale that quantifies progressive steps in sleepiness, ranging from a score of 1 denoting high alertness to a score of 7 indicating imminent sleep.

Following this, a battery of four reaction time tests of increasing difficulty was administered. This sequence introduced progressively more complex elements of working memory scanning and divided attention. ${ }^{13}$ The method of test presentation and analysis on a personal computer followed the techniques of Miller and colleagues ${ }^{13}$ and Aguso-Mateos and colleagues ${ }^{14}$ with minor modifications. Each test consisted of five practice trials to enhance the stability of performance by reducing the learning effect, ${ }^{15}$ immediately followed by the test proper consisting of 30 trials. The battery took approximately 35 minutes to complete, an appropriate length to detect the effects of moderate sleep loss. ${ }^{16}$

The RT targets consisted of single digits between 0 and 9 presented in a box in the centre of a 17-inch computer monitor. The height of the digits was approximately $6 \mathrm{~mm}$, and at a viewing distance of $60 \mathrm{~cm}$, subtended roughly $34^{\prime}$ at the retina. Subjects were requested to press the spacebar of the keyboard as quickly as possible with their preferred hand, following the appearance of a target, but to make as few errors as possible. Targets appeared on the monitor for one second. Failure to respond within this period resulted in the trial being classified as "missed". Key presses during intertrial intervals or responses to a non-target were classified as "invalid" responses. The four RT tests were as follows:

(1) Simple RT. Subjects were required to respond to the appearance of any digit. The inter-target interval was randomised within the range 1.5-7.5 seconds.

(2) Complex RT. The target was a specified digit, the others to be ignored by the subject, requiring an element of response inhibition.

(3) Sequential RT 1. This task required the recognition of the repetition of a digit in two consecutive trials. Subjects had to respond to the second digit in the sequence, thereby including a short term memory component.

(4) Sequential RT 2. In a slightly more difficult task to that in the preceding test, subjects had to respond to the second of two consecutive digits appearing in ascending order.

The inter-trial interval in tests 2-4 was one second. The proportion of targets to non-targets was approximately 1:5, with 3-8 non-targets interposed between each target.

The mean RT for each test was calculated for up to 26 trials after the exclusion of the two longest and two shortest individual reaction times. Missed and invalid responses were combined to provide an accuracy score, expressed as a percentage, for each of the four tests.

Change scores were the percentage differences between the after night duty and baseline assessments. A deterioration of $>15 \%$ was considered of practical importance on the basis of reported $8-15 \%$ increases in a range of psychomotor tests, including simple RT, corresponding to a blood alcohol level of $0.05 \% .^{10}$

\section{Statistical analysis}

The vector of RT test parameters was tested with Hotelling's $\mathrm{T}^{2}$ test. Subsequently for each of the reaction time parameters the outcomes before and after night duty were compared using a random effects time series regression model adjusting for gender, age, extent of sleep loss, and change in subjective sleepiness. Along with summary statistics and p values, 95\% confidence intervals (CI) were also reported. All testing was done at the 0.05 level of significance.

\section{RESULTS}

The mean duration of sleep on the baseline night preceding night duty was 7.04 hours (SD 1.20) which did not differ significantly from the reported mean habitual sleeping time of 7.02 hours (SD 0.54) (Student's paired $t$ test: $\mathrm{p}=0.92$ with $95 \%$ CI for difference -0.47 to 0.42 ). The mean amount of sleep during night duty was 1.66 hours (SD 1.40), significantly less than baseline nocturnal sleep (Student's paired $t$ test: $\mathrm{p}<0.001$ with $95 \%$ CI for difference -6.05 to -4.71 ). Only three subjects reported sleeping for more than three hours during the night duty period.

At baseline all the subjects rated their degree of sleepiness between 1 and 3 points on the Stanford Sleepiness Scale (SSS). The mean increase in SSS score after night duty was 2.0 points (95\% CI for difference 1.7 to 2.2 ).

Hotelling's $\mathrm{T}^{2}$ test results revealed that the vector of change in response times was significantly different from the zero vector $(p=0.0045)$. The same analysis of the vector of change in response accuracy provided similar results $(p=0.0074)$. In particular, from the univariate random effects time series regression the response times in tests 1 (simple RT), 2 (choice RT), and 3 (sequence RT 1) were significantly prolonged after night duty (baseline value compared to after night duty value, $\mathrm{p}<0.005$ ) (table 1 ). The same trend applied to test 4 (sequence RT 2), but the difference failed to attain significance $(p=0.08)$. Figure 1 illustrates these findings. Concerning response accuracy, significant deterioration occurred after night duty in tests 3 and 4 , while in tests 1 and 2 the change was not significant (table 1). The highest mean deterioration in response speed was $12 \%$ in test 1 . The remaining tests had a mean deterioration of $6-7 \%$. The highest mean decline in response accuracy was in test $4(6 \%)$; it was $2 \%$ in test 3 and $<1 \%$ in the first two tests.

Table 2 shows the number of subjects whose response speed (RT) or accuracy of response deteriorated by $>15 \%$ in respect of the four tests. Overall 17/33 (52\%, 95\% CI 34.5\% to $68.6 \%$ ) deteriorated by $>15 \%$ on at least one of the eight RT measures.

\section{DISCUSSION}

The main finding of this study is that sleep deprivation, associated with night duty, induced important psychomotor deficits likely to be of occupational importance in 52\% of a group of resident anaesthetists.

Our baseline group mean response time scores increased progressively with task complexity as anticipated from previous results involving patient groups. ${ }^{13}{ }^{14}$ After night duty this trend was maintained, but with significantly longer response times except for the fourth test, the most complex of the series. While accuracy was maintained in the $99 \%$ range 
Table 1 Change in reaction time (RT) test results from baseline to after night duty

\begin{tabular}{|c|c|c|c|c|}
\hline Tests & Baseline mean (SD) $[95 \% \mathrm{Cl}]$ & After duty mean (SD) $[95 \% \mathrm{Cl}]$ & $z$ test $p$ value & $95 \% \mathrm{Cl}$ for difference* \\
\hline \multicolumn{5}{|l|}{ (1) Simple RT } \\
\hline $\mathrm{RT}(\mathrm{ms})$ & 315.3 (24.3) [306.7 to 324.0$]$ & $351.5(56.0)$ [331.7 to 371.4$]$ & $<0.001$ & -54.9 to -17.4 \\
\hline $\begin{array}{l}\text { Accuracy (\%) } \\
\text { (2) Choice RT }\end{array}$ & $99.4(1.52)[98.9$ to 100.0$]$ & 98.7 (4.19) [97.2 to 100.0$]$ & 0.31 & -2.03 to 0.64 \\
\hline RT (ms) & 405.9 (38.5) [392.2 to 419.5 ] & 430.9 (45.4) [414.8 to 447.0$]$ & $<0.001$ & -37.7 to -12.3 \\
\hline $\begin{array}{l}\text { Accuracy (\%) } \\
\text { (3) Sequence RT } 1\end{array}$ & $99.8(0.42)[99.6$ to 99.9$]$ & $99.2(1.88)[98.5$ to 99.9$]$ & 0.08 & -1.23 to 0.08 \\
\hline RT (ms) & 439.1 (58.0) [418.6 to 459.7 ] & $467.3(76.6)$ [440.1 to 494.5$]$ & 0.004 & -47.1 to -9.2 \\
\hline $\begin{array}{l}\text { Accuracy (\%) } \\
\text { (4) Sequence RT } 2\end{array}$ & $99.5(0.71)$ [99.2 to 99.7] & $97.3(5.16)$ [95.5 to 99.2] & 0.01 & -3.82 to -0.48 \\
\hline RT (ms) & $465.5(76.2)[437.5$ to 493.4$]$ & 487.1 (89.8) [455.3 to 519.0] & 0.08 & -48.3 to 2.9 \\
\hline Accuracy (\%) & $98.3(1.90)[97.6$ to 99.0$]$ & $92.7(8.32)[89.7$ to 95.6$]$ & $<0.001$ & -8.31 to -3.00 \\
\hline
\end{tabular}

in the two more simple tasks, statistically significant declines in accuracy after night duty were found in the two more complex tasks. This conventional analysis is largely in accordance with some previous RT findings. ${ }^{8}$ However, both simple and more complex RT tasks were significantly affected by sleep loss in our study contrasting with the findings of Orton and Gruzelier, ${ }^{7}$ possibly due to the differences in the length of the tests used.

Consideration of group percentage changes between baseline and after sleep deprivation revealed 6-12\% mean prolongation of response times, the highest occurring in respect of simple RT. The extent of deterioration in simple RT is relatively close to the $9 \%$ in a previous study after 22 hours of continuous wakefulness in non-medical personnel. ${ }^{10}$ These findings concur with the view that simple, monotonous tasks are most susceptible to deterioration of response time while more challenging tasks may be less affected. ${ }^{16}$

Aspects of accuracy in a simple RT task were reported to show a comparable sensitivity to that of speed measures after sleep deprivation in non-medical personnel. ${ }^{10}$ This was not the case in our study where mean accuracy for simple RT remained at high levels (above 98\%) following night duty. Nonetheless, there was a clear trend in our data of decreasing accuracy scores with increasing task complexity. Statistically significant mean decrements occurred in respect of the two more difficult tasks.

The assessment of individual changes after sleep loss relative to the reported equivalent effects of alcohol ingestion adds another dimension to this study. The relevance of the BAC equivalent criterion for sleep deprivation effects has been alluded to in the medical context. ${ }^{2} 1718$ The chosen criterion in the present study was based on the reported 8$15 \%$ deterioration in response speed in a range of RT and vigilance tasks induced by a BAC of $0.05 \%$. In respect of simple RT the deterioration was $9 \% .^{10}$ The chosen cut-off of $>15 \%$ deterioration was applied to each of our four tasks and is possibly on the conservative side. While $30 \%$ of our subjects exceeded $15 \%$ deterioration in response speed in simple RT

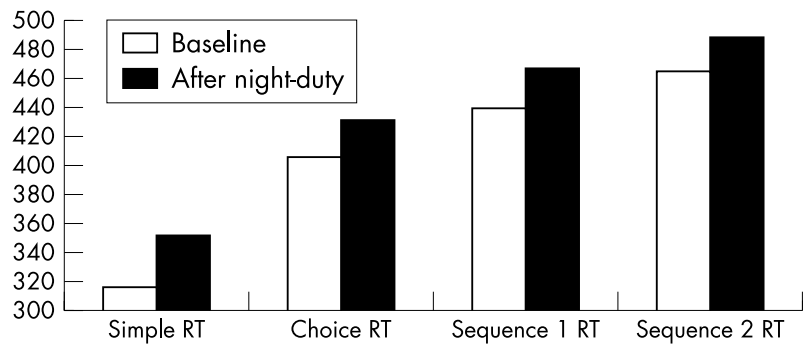

Figure 1 Mean reaction time ( $\mathrm{ms}$ ) for each of the four tests in respect of baseline and after night duty assessments. and in the first of two tests of sequence RT, $49 \%$ of the group deteriorated in respect of at least one of the four speed measures. An important decline in response accuracy occurred in far fewer subjects. Nevertheless, as task complexity increased, so did the number of subjects with an important degree of deterioration. While only one subject deteriorated by $>15 \%$ in respect of either simple or choice RT, $4 / 31$ subjects $(12.9 \%)$ deteriorated on the most complex test, the second of the sequence RT tasks. Overall $52 \%$ of subjects deteriorated in terms of at least one response speed or accuracy measure. On the other hand a subgroup of $18 \%$ of subjects evinced resistance to sleep deprivation in terms of the performance measures employed in this study by virtue of a deterioration of $<8 \%$ on any of the test measures. If these marked inter-individual differences in susceptibility to sleep deprivation are confirmed in subsequent studies they could conceivably influence policy decisions.

The subjects in our study were exposed to a relatively benign work programme in terms of the frequency of night duty (once per week) and in the total number of hours worked per week (mean 60.5 hours) in contrast to those in many previous studies. ${ }^{19}$ This is likely to have reduced the risk of chronic partial sleep deprivation, but the effects of sleep loss were still detectable in a sizeable proportion of our subjects.

The dichotomised outcomes of the response time measures of tests 1-4 were also modelled against age, gender, degree of sleep deprivation while on duty, and self rated sleepiness. For some tests the number of subjects appeared insufficient to render stable estimates for the odds ratios and their 95\% CIs. Despite the lack of statistical significance there was a trend in the test of simple RT and in the two sequence RT tests for an increase in age to be protective against sleep deprivation.

Table 2 The percentage of subjects who deteriorated by more than $15 \%$ from baseline to after night duty

\begin{tabular}{|c|c|c|}
\hline Tests & $\begin{array}{l}>15 \% \text { deterioration, } \\
\% \text { subjects (proportion) }\end{array}$ & $95 \% \mathrm{Cl}(\%)$ \\
\hline \multicolumn{3}{|l|}{ (1) Simple RT } \\
\hline Time & $30.3 \%(10 / 33)$ & 14.6 to 46.0 \\
\hline $\begin{array}{l}\text { Accuracy } \\
\text { (2) Choice RT }\end{array}$ & $3.0 \%(1 / 33)$ & 0.0 to $8.9^{*}$ \\
\hline Time & $9.1 \%(3 / 33)$ & 0.0 to $18.9^{*}$ \\
\hline $\begin{array}{l}\text { Accuracy } \\
\text { (3) Sequence RT I }\end{array}$ & $0.0 \%(0 / 33)$ & \\
\hline Time & $30.3 \%(10 / 33)$ & 14.6 to 46.0 \\
\hline $\begin{array}{l}\text { Accuracy } \\
\text { (4) Sequence RT } 2\end{array}$ & $6.1 \%(2 / 33)$ & 0.0 to $14.2^{*}$ \\
\hline Time & $22.6 \%(7 / 31)$ & 7.9 to 37.3 \\
\hline Accuracy & $12.9 \%(4 / 31)$ & 1.1 to 24.7 \\
\hline
\end{tabular}

*When the lower limit of the $95 \% \mathrm{Cl}$ was found to be negative 0.0 was used. 
Similarly female gender appeared protective against deterioration on simple RT and the second sequence RT test. The majority of our subjects (25/33) obtained some sleep during the course of their duty. In all cases this was in the early hours of the morning before 0700 and within a few hours of the testing session. Sleeping for $<1$ hour during night duty suggested increased risk for a poor outcome in choice RT and in both the sequence RT tasks. This finding requires confirmation before it is regarded as contradicting several previous studies showing that brief periods of sleep during night duty offered little or no protection from the effects of sleep deprivation. ${ }^{11}$ Wilkinson ${ }^{20}$ contended that significant decreases in vigilance performance follow a night in which sleep is restricted to three hours or less. Richardson and colleagues ${ }^{11}$ found that the provision of protected four hour periods for residents to sleep during duty did not significantly improve performance on a 15 minute vigilance task. Partial sleep deprivation may have a greater negative effect on functioning than either long term or short term total sleep deprivation. ${ }^{1}$

Inconsistent performance across tests was apparent in our results. For instance, only 5/17 subjects deteriorated in three or four of the tests while 10/17 deteriorated in one task only. These findings support the proposal that sleep deprivation causes state instability characterised by fluctuating levels of attention and alertness. ${ }^{21} 22$

We took cognisance of the methodological criticism of previous studies. ${ }^{3}$ However, we did not comply with the recommendation to counterbalance the order of testing. Practical difficulties were caused by the frequent relocation of our subjects to hospitals over a considerable geographical area. Compliance with this recommendation would have necessitated greater separation in the timing of the baseline and after-night duty assessments, thereby increasing the rate of attrition. The possibility of diluting the effects of sleep deprivation by a practice effect in the psychomotor assessments was thus unavoidable. This effect is likely to have been slight by virtue of the relative simplicity of the tasks, each of which was preceded by practice trials, and the highly educated subjects.

In conclusion, this study revealed significant performance deficits in a sizeable proportion of residents in a relatively benign programme in terms of the frequency of night duty and in the number of hours worked per week. The likelihood of this impinging on the real-life monitoring ability of anaesthetists should not be ignored.

\section{Authors' affiliations}

P Bartel, Department of Neurology, University of Pretoria and Pretoria Academic Hospital, South Africa
W Offermeier, F Smith, Department of Anaesthesiology, University of Pretoria and Pretoria Academic Hospital, South Africa

P Becker, Biostatistics Unit, South African Medical Research Council, Pretoria, South Africa

Presented in part at the joint congress of the Association of British Neurologists and the Neurological Association of South Africa, 29 Jan1 Feb 2003, Cape Town, South Africa

\section{REFERENCES}

1 Pilcher JJ, Huffcutt Al. Effects of sleep deprivation on performance: a metaanalysis. Sleep 1996;19:318-26.

2 Feyer A-M. Fatigue: time to recognise and deal with an old problem. BMJ 2001;322:808-9.

3 Weinger MB, Ancoli-Israel S. Sleep deprivation and clinical performance. JAMA 2002;287:955-7.

4 Samkoff JS, Jacques CHM. A review of studies concerning effects of sleep deprivation and fatigue on residents' performance. Acad Med 1991;66:687-93.

5 Harrison Y, Horne JA. The impact of sleep deprivation on decision making: a review. J Exp Psychol Appl 2000;6:236-49.

6 Narang V, Laycock JRD. Psychomotor testing of on-call anaesthetists. Anaesthesia 1986;41:868-9.

7 Orton DI, Gruzelier JH. Adverse changes in mood and cognitive performance of house officers after night duty. BMJ 1989;298:21-3.

8 Wesnes KA, Walker MB, Walker LG, et al. Cognitive performance and mood after a weekend on call in a surgical unit. Br J Surg 1997;84:493-5.

9 Dawson D, Reid K. Fatigue, alcohol and performance impairment. Nature 1997;388:235

10 Williamson AM, Feyer A-M. Moderate sleep deprivation produces impairments in cognitive and motor performance equivalent to legally prescribed levels of alcohol intoxication. Occup Environ Med 2000;57:649-55.

11 Richardson GS, Wyatt JK, Sullivan JP, et al. Objective assessment of sleep and alertness in medical housestaff and the impact of protected time for sleep. Sleep 1996;19:718-26.

12 Hoddes E, Zarcone V, Smythe H, et al. Quantification of sleepiness: a new approach. Psychophysiology 1973;10:431-6.

13 Miller EN, Satz P, Visscher B. Computerized and conventional neuropsychological assessment of HIV-1-infected homosexual men. Neurology 1991;41:1608-16.

14 Ayuso-Mateos JL, Pereda M, Gmez del Barrio A, et al. Slowed reaction time in HIV-1-seropositive intravenous drug users without AIDS. Eur Neurol 2000;44:72-8.

15 Casagrande $M$, Violani C, Curcio G, et al. Assessing vigilance through a brief pencil and paper letter cancellation task (LCT): effects of one night of sleep deprivation and of the time of day. Ergonomics 1997;40:613-30.

16 Wilkinson RT. The measurement of sleepiness. In Broughton RJ, Ogilvie RD, eds. Sleep, arousal and performance. Boston: Birkhauser, 1992:254-65.

17 Lichtor JL, Zacny J, Lane BS, et al. Research grant report: how does night call affect next-day performance in anesthesia? J Clin Monit 1996;12:277-8.

18 Kuo AA. Resident overnight call-an idea past its time? West J Med $2001 ; 174: 180-1$.

19 Owens JA, Veasey SC, Rosen RC. Physician, heal thyself: sleep, fatigue, and medical education. Sleep $2001 ; 24: 493-5$.

20 Wilkinson RT. Effects of up to 60 hours sleep deprivation on different types of work. Ergonomics 1964;7:175-86.

21 Koslowsky M, Babkoff $\mathrm{H}$. Meta-analysis of the relationship between total sleep deprivation and performance. Chronobiol Int 1992;9:132-6.

22 Doran SM, Van Dongen HP, Dinges DF. Sustained attention performance during sleep deprivation: evidence of state instability. Arch Ital Biol $2001 ; 139: 253-67$ 